\title{
Use of a flexible hollow-core carbon dioxide laser for microsurgical resection of vestibular schwannomas
}

\author{
Marc S. Schwartz, MD, ${ }^{1}$ and Gregory P. Lekovic, MD, PhD² \\ 'Department of Neurosurgery, University of California, San Diego; and ${ }^{2}$ Neurosurgery, House Clinic, Los Angeles, California
}

OBJECTIVE The $\mathrm{CO}_{2}$ laser has been used on an intermittent basis in the microsurgical resection of brain tumors for decades. These lasers were typically cumbersome to use due to the need for a large, bulky design since infrared light cannot be transmitted via fiber-optic cables. Development of the OmniGuide cable, which is hollow and lined with an omnidirectional dielectric mirror, has facilitated the reintroduction of the $\mathrm{CO}_{2}$ laser in surgical use in a number of fields. This device allows for handheld use of the $\mathrm{CO}_{2}$ laser in a much more ergonomically favorable configuration, holding promise for microneurosurgical applications. This device was introduced into the authors' practice for use in the microsurgical resection of skull base tumors, including vestibular schwannomas.

METHODS The authors reviewed the initial 41 vestibular schwannomas that were treated using the OmniGuide $\mathrm{CO}_{2}$ laser during an 8-month period from March 2010 to October 2010. The laser was used for all large tumors, and select medium-sized tumors were treated via both the translabyrinthine and retrosigmoid approaches. The estimated time of tumor resection and estimated blood loss were obtained from operating room records. Data regarding complications, facial nerve and hearing outcomes, and further treatment were collected from hospital and clinic records, MRI reports, and direct review of MR images. Time of resection and blood loss were compared to a control group $(n=18)$ who underwent surgery just prior to use of the laser.

RESULTS A total of 41 patients with vestibular schwannomas were surgically treated. The median estimated time of tumor resection was 150 minutes, and the median estimated blood loss was $300 \mathrm{ml}$. The only operative complication was 1 CSF leak. Thirty-eight patients had normal facial nerve function at late follow-up. The median MRI follow-up was 52 months, and, during that time, only 1 patient required further treatment for regrowth of a residual tumor.

CONCLUSIONS The OmniGuide $\mathrm{CO}_{2}$ laser is a useful adjunct in the resection of large vestibular schwannomas. This device was used primarily as a cutting tool rather than for tumor vaporization, and it was found to be of most use for very large and/or firm tumors. There were no laser-associated complications, and the results compared favorably to earlier reports of vestibular schwannoma resection. https://thejns.org/doi/abs/10.3171/2017.12.FOCUS17592

KEY WORDS carbon dioxide laser; vestibular schwannoma; acoustic neuroma; microsurgery; omnidirectional dielectric mirror

$\mathrm{L}$ ASERS have been used in neurosurgery for decades. Stellar et al. reported use of a $\mathrm{CO}_{2}$ laser for resection of an intracranial tumor in $1970 .{ }^{34}$ The rationale for laser use in tumor resection is both to allow for "no-touch" cutting and for tissue debulking, with hemostatic benefit. The $\mathrm{CO}_{2}$ laser has particular advantages in surgery. Its infrared wavelength $(10.6 \mu \mathrm{m})$ penetrates water very poorly, confining its area of action to the surface of the biological structures being dissected and minimizing collateral tissue damage..$^{5,29}$

The benefits of the $\mathrm{CO}_{2}$ laser, however, have been tempered by ergonomic and practical difficulties. ${ }^{28}$ All materials usable for fiber-optic transmission in the infrared spectrum at $10.6 \mu \mathrm{m}$ are opaque to light. ${ }^{36}$ Thus, fiberoptic cables cannot be used. Previously, $\mathrm{CO}_{2}$ laser energy could only be transmitted through a housing with mirrors

ABBREVIATIONS AAO-HNS = American Academy of Otolaryngology-Head and Neck Surgery; CPA = cerebellopontine angle; EBL = estimated blood loss; ETR = elapsed time of tumor resection; H-B = House-Brackmann; RS = retrosigmoid; TL = translabyrinthine.

SUBMITTED September 11, 2017. ACCEPTED December 7, 2017.

INCLUDE WHEN CITING DOI: 10.3171/2017.12.FOCUS17592. 
and bulky articulating arms. While used successfully, including for microsurgical resection of vestibular schwannomas, practical limitations prevented widespread adoption. ${ }^{26}$

Development of a hollow fiber, lined internally with an omnidirectional dielectric mirror, allows for transmission of a $\mathrm{CO}_{2}$ laser through an easily manipulatable cable. ${ }^{12}$ When attached to an ergonomically favorable dissector, practical difficulties of $\mathrm{CO}_{2}$ laser use for intracranial microneurosurgery can be minimized. ${ }^{20}$ We present our series of case experience using the OmniGuide (ARC Laser $\mathrm{GmbH}) \mathrm{CO}_{2}$ laser for microsurgical resection of vestibular schwannomas.

\section{Methods}

An institutional review board waiver was obtained prior to review of any data for this study. During an 8-month period from March 2010 to October 2010, the OmniGuide $\mathrm{CO}_{2}$ laser was used by the first author as an adjunct in the resection of medium to large vestibular schwannomas. All operations were carried out by a team consisting of a single neurosurgeon and one of 6 neurotologists. For large tumors $(>2.5 \mathrm{~cm}$ in the cerebellopontine angle [CPA]), the laser was used for all consecutive cases with the exception of a single, very soft, cystic tumor. For medium tumors $(1.5-2.5 \mathrm{~cm}$ in the CPA), the laser was used for selected cases on an ad hoc basis. All consecutive cases of laser use are reported.

\section{Outcome Measures}

Given that the primary benefits of $\mathrm{CO}_{2}$ laser usage were thought to be related to operative time and blood loss, we chose elapsed time of tumor resection (ETR) and estimated intraoperative blood loss as primary outcome measures. Data regarding these measures were obtained retrospectively through review of the hospital chart. ETR was determined from the facial nerve monitoring record, in which the times of "neurosurgeon begins" and "tumor out" were recorded. Of note, for retrosigmoid cases this included drilling of the porus and exposure of the intracanalicular portion of the tumor. Estimated blood loss (EBL) was obtained from the anesthesia record and could only be determined for the entire procedure.

Secondary outcome measures were obtained from a prospectively generated database in a manner suitable for statistical analysis. These measures included facial nerve function, which was graded from I to VI according to the House-Brackmann (H-B) scale, and hearing, which was categorized as per the American Academy of Otolaryngology-Head and Neck Surgery (AAO-HNS) classification system. ${ }^{17,22}$ Complications, totality of tumor resection, MRI results, and the need for subsequent treatment were also recorded.

\section{Patient Population}

A total of 41 patients were surgically treated in this series. Demographic data are presented in Table 1. Patients with neurofibromatosis type 2 and those who had previously been treated with stereotactic radiosurgery were included. No patient who underwent surgery during the
TABLE 1. Characteristics of 41 patients undergoing surgery for vestibular schwannoma using the OmniGuide $\mathrm{CO}_{2}$ laser

\begin{tabular}{cc}
\hline \multicolumn{1}{c}{ Characteristic } & Value \\
\hline Age in yrs & \\
\hline Mean (SD) & $48.3(15.2)$ \\
\hline Median (range) & $47(19-95)$ \\
\hline Sex, \% M/F & $53.7 / 46.3$ \\
\hline Side, $\%$ rt/lt & $58.5 / 41.5$ \\
\hline Total tumor size in cm & $3.1(0.8)$ \\
\hline Mean (SD) & $3.1(1.8-5.3)$ \\
\hline Median (range) & $2.8(0.9)$ \\
\hline CPA tumor size & $2.7(1.5-5.3)$ \\
\hline Mean (SD) & $58.5 / 41.5$ \\
\hline Median (range) & $15(36.6)$ \\
\hline Tumor size, \% large/medium & $4(9.8)$ \\
\hline Preop AAO-HNS hearing class, $\mathrm{n}(\%)$ & $1(2.4)$ \\
\hline A & $21(51.2)$ \\
\hline B & $5(12.2)$ \\
\hline C & $4(9.8)$ \\
\hline D
\end{tabular}

study period had previously undergone microsurgical resection. In addition, a representative control group of 18 patients who had undergone surgery immediately prior to the study period without the use of the laser were included for analyses regarding blood loss and operative time. The mean age at surgery, total tumor size, and size of tumor extension into the CPA did not differ significantly between the control group and the OmniGuide group. The control group appeared to have a greater prevalence of mediumsized tumors than the OmniGuide group $(61.1 \%$ vs $41.5 \%$, respectively). This difference did not achieve statistical significance $(\mathrm{p}=0.16)$.

All large tumors were approached via the translabyrinthine (TL) route. The retrosigmoid (RS) approach was reserved for patients with medium-sized tumors and good hearing. Patients with good hearing and with tumors small enough and configured so as to provide a reasonable chance of hearing preservation were offered the option of the RS approach. The decision about which operation to perform was made on a case-by-case basis. The rate of use of the RS approach was similar in the OmniGuide and control groups (9.8\% and $11.1 \%$, respectively).

\section{Procedures}

Operative techniques of both TL and RS approaches to resection have previously been described in detail. ${ }^{10,32} \mathrm{TL}$ procedures were carried out in the supine position with the patient's head freely positioned on the operating table in contralateral rotation. RS procedures were carried out in the lateral park-bench position with the patient's head immobilized using a Mayfield device (Integra LifeSciences). Continuous facial nerve monitoring using the XomedNIM system (Medtronic) was used for all cases. For cases 

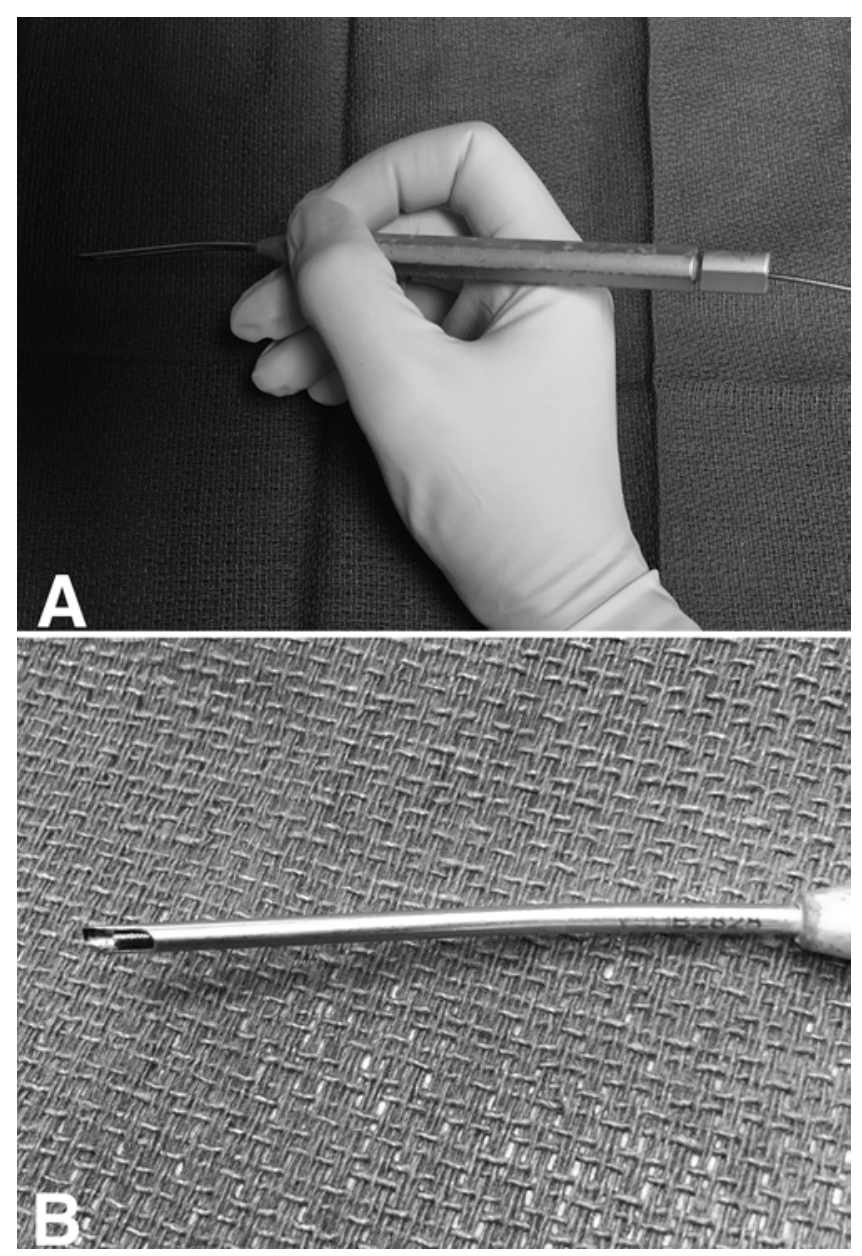

FIG. 1. Photographs of the OmniGuide cable and handpiece. A: The tip of the handpiece also functions as a microdissector, facilitating tissue manipulation and ensuring proper positioning. B: When properly set up, the end of the cable rests in the midpoint of the oval-shaped aperture at the dissector tip.

of attempted hearing preservation, evoked auditory brainstem responses were recorded continuously (Cadwell Cascade, Cadwell Industries). In most cases, we begin surgery with the goal of gross-total tumor resection. If the tumor is found to be highly adherent to the facial nerve, or if facial nerve function is otherwise in jeopardy, we prioritize the facial nerve over gross-total tumor resection. Our philosophy regarding aggressiveness of tumor resection has been described. ${ }^{13,31}$

After tumor resection, patients were observed in the intensive care unit overnight and began mobilization on the 1st postoperative day. Patients were discharged from the hospital after meeting discharge criteria, including tolerance of diet and safe ambulation. Facial nerve function was assessed immediately postoperatively and throughout the postoperative period. Any patient who had not experienced any facial weakness as of 1 month postoperatively was deemed to have an ultimate result of normal facial nerve function (H-B grade I). For patients with any postoperative facial weakness, late facial nerve function was determined at 1 year. Postoperative audiograms were obtained in all patients who underwent $\mathrm{RS}$ resection.
As per our usual practice, MRI was not performed in the immediate postoperative period unless there were specific clinical indications. A schedule for postoperative imaging was determined individually for each. A first postoperative scan was generally recommended at some point between 4 and 12 months after surgery.

\section{Device Description}

The OmniGuide $\mathrm{CO}_{2}$ laser system consists of a laser source generator, a flexible hollow-core fiber, and a handheld dissector housing the end of the fiber (Fig. 1). We used the OmniGuide FELS-25A laser (ARC Laser GmbH) using the continuous mode. Power output was limited to the $20-\mathrm{W}$ maximum specified for the OmniGuide fiber. Flow of helium through the cable acts as a coolant and as the gaseous substrate. This ensures that the laser wavelength is calibrated to the cable's omnidirectional dielectric mirror, allowing for proper propagation of the laser energy.

We used the Beam Path Neuro-L fiber (OmniGuide), which has an outer diameter of $1.2 \mathrm{~mm}$. At the operative end of the cable, the laser energy is defocused, resulting in decreasing intensity proportional to the square of the distance from the fiber tip. The end of the fiber fits within a rigid handpiece, allowing for manipulation within a deep corridor. We used the Neuro-HP-5 medium length, straight handpiece in all cases (OmniGuide).

\section{Data Analysis}

Descriptive statistics, including mean, standard deviation, median, minimum, and maximum values, were computed for all interval-level variables and frequency distribution for all categorical variables. The OmniGuide and control groups were compared using independent group t-tests (after confirmation of homogeneity of variance) for interval-level data and chi-square or Fisher's exact test for categorical variables.

\section{Results}

\section{Operative Technique}

The flexible hollow-core $\mathrm{CO}_{2}$ laser was used as an adjunct to tumor resection rather than as the only tool. The OmniGuide Neuro-HP-5 handpiece was ergonomically well suited to visibility and surgical manipulation in all cases. Laser power can be adjusted in 2-W increments, and a setting of $12 \mathrm{~W}$ was found to be generally most useful for bulk tumor resection. Lower settings, in the 6- to $10-\mathrm{W}$ range, were useful for more delicate work, especially in proximity to cranial nerves.

We quickly realized that the laser functioned best as a cutting tool rather than a vaporizing tool. As is our usual method, central tumor debulking was carried out primarily using an ultrasonic aspirator. After debulking, the periphery of the tumor could be dissected from surrounding structures and then further removed in a piecemeal fashion using the laser, with the tip of the device held very close to tumor tissue (Fig. 2). The laser was found to have several advantages over microscissors. First, it could cut through even the firmest tissue, including portions of tumors that were not amenable to division using metal scissors. Second, tumor could be cut while simultaneously being re- 


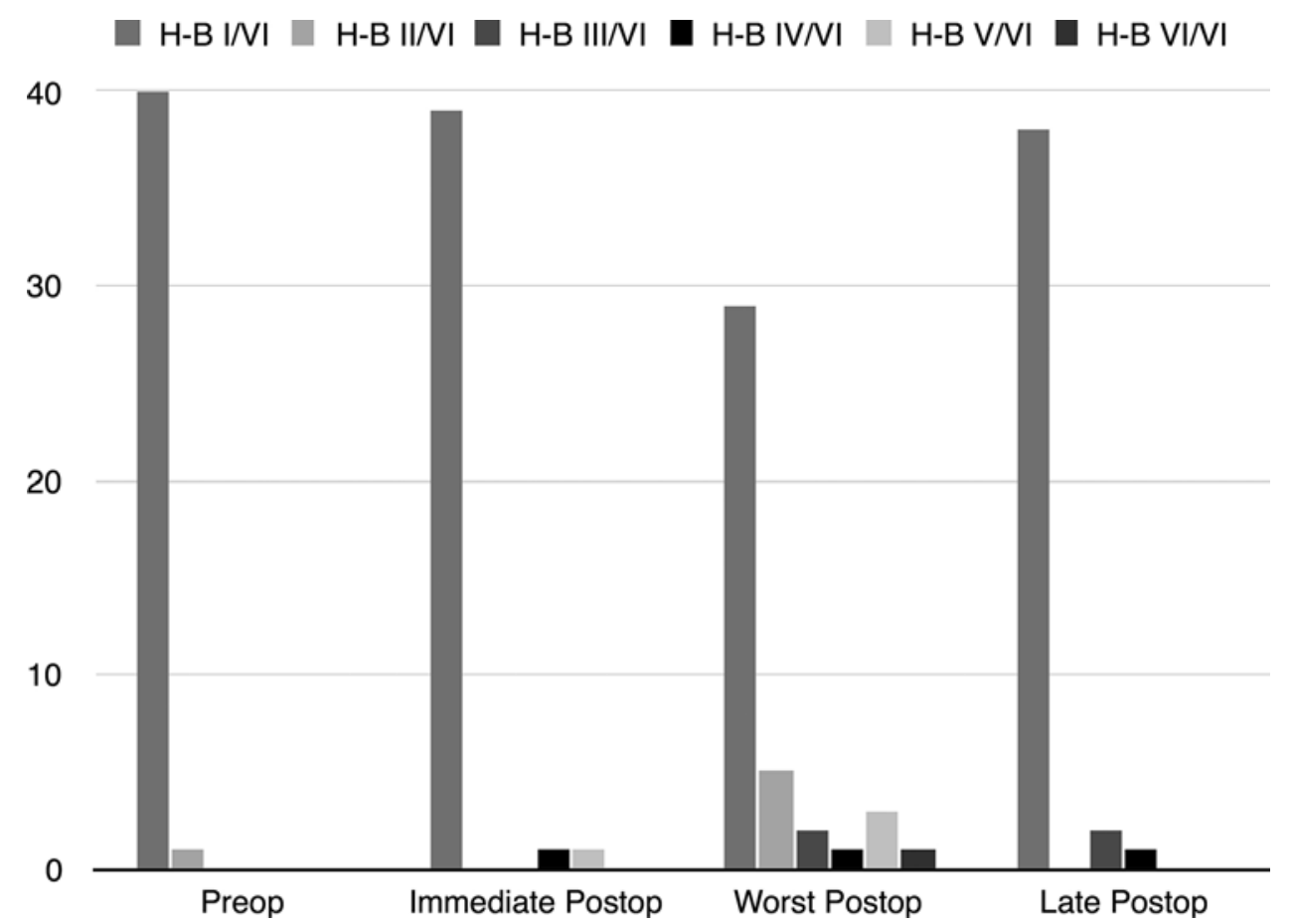

FIG. 2. Bar graph showing facial nerve results. Of the 41 patients who underwent surgery, 12 had some degree of temporary facial weakness; 38 had normal facial nerve function at late follow-up.

tracted centrally by a suction device held in the nondominant hand. This avoided the typical pushing action of microscissors and allowed for the resection of larger pieces of tumor in fewer steps. Third, cutting tumor always resulted in some vaporization, further speeding resection. Finally, while the hemostatic action of the $\mathrm{CO}_{2}$ laser on larger vessels was minimal, small vessels were easily cauterized, resulting in the perception of less ongoing blood loss.

To prevent the potentially dangerous action of the laser at a distance, we were careful to always point the laser toward the center of the tumor mass so that when tissue was cut through, the ongoing beam was still confined within the tumor. Furthermore, we were careful to always keep the depths of the field bathed in saline solution, thus protecting submerged structures. As the laser output is defocused, objects distant from the tip of the laser were also relatively protected from laser energy, proportional to the square of the distance, in any.

The $\mathrm{CO}_{2}$ laser was also used to debulk tumor more delicately using a no-touch technique in areas in which cranial nerve traction was a concern. Typically, this was close to the porus of the internal auditory canal. When working near cranial nerves, we took care to ensure that these structures remained irrigated. Except when the laser was used at the lowest power, small fragments of char could be seen floating away from the site of cutting. While there was no evidence that these fragments deposited any significant heat, we typically protected the facial nerve with a moist cotton patty when working nearby. The OmniGuide laser was never used for dissection of tumor tissue directly from cranial nerves or other vital structures.

The laser fiber proved to be fragile under certain cir- cumstances. Use at a power greater than $12 \mathrm{~W}$ for prolonged periods was not practical due to device failure. This problem may have since been corrected as a result of improvements in manufacturing. Furthermore, obstruction of the aperture at the outlet of the cable occasionally led to failure due to overheating in the absence of flowing helium. Finally, any kinking of the fiber always resulted in breakage. Our operative technique is demonstrated and discussed further in the accompanying Video 1.

VIDEO 1. Brief video demonstrating the use of the OmniGuide $\mathrm{CO}_{2}$ laser as an adjunct to resection of a large vestibular schwannoma via the translabyrinthine route. Gross-total tumor resection was achieved, and the patient's facial nerve function remained normal. Copyright Marc S. Schwartz. Published with permission. Click here to view.

\section{Operative Time and Blood Loss}

The median ETR for the OmniGuide patients was 150 minutes (mean 152.1 minutes, SD 61.8 minutes, range 64-333 minutes) and did not differ significantly from that of the control group (median 105 minutes, mean 129.7 minutes, SD 63.7 minutes, range 62-283 minutes), which had a similar range and variability in resection time. The median EBL was $300 \mathrm{ml}$ (mean $351.2 \mathrm{ml}$, SD $155.9 \mathrm{ml}$, range $200-1000 \mathrm{ml}$ ), which is also not significantly different from that of the control group (median $300 \mathrm{ml}$, mean $338.9 \mathrm{ml}$, SD $113.4 \mathrm{ml}$, range $200-650 \mathrm{ml})$. No patient in either group required transfusion of blood products.

\section{Facial Nerve, Hearing, and Complications}

Facial nerve outcomes are detailed in Fig. 2. Preoperatively, only 1 patient (2.4\%), who had a giant tumor, had 

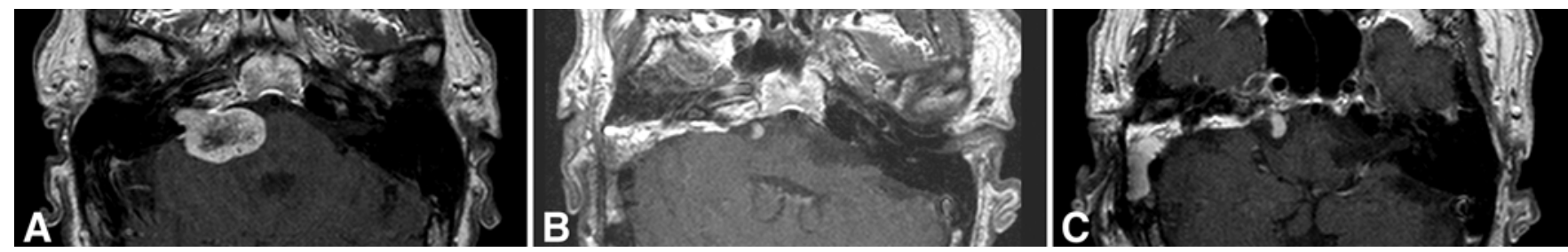

FIG. 3. A: Preoperative axial, enhanced T1-weighted MR image obtained in a patient who later underwent Gamma Knife radiosurgery for growing residual tumor. B: First postoperative MR image showing a small residual tumor. C: Later MR image demonstrating growth of a residual tumor.

facial weakness. This mild weakness improved to normal postoperatively. Immediately following surgery, 2 patients (4.9\%) had new facial weakness. Ten additional patients developed some degree of delayed facial weakness in the days after surgery (total of 12 patients [29.3\%]). Most of these resolved completely. Ultimately, $38(92.7 \%)$ patients had normal facial nerve function. Two patients $(4.9 \%)$ were left with H-B grade III function and 1 (2.4\%) with $\mathrm{H}-\mathrm{B}$ grade IV function.

Of the 4 patients undergoing attempted hearing preservation surgery with the use of the $\mathrm{CO}_{2}$ laser via the RS route, $2(50 \%)$ had preserved hearing at the preoperative level (1 each in class A and class B) and 2 lost hearing entirely.

One patient (2.4\%) developed CSF rhinorrhea postoperatively, which was successfully treated by blind sac closure of the external auditory canal and direct packing of the eustachian tube. ${ }^{11}$ This was the only complication. Excepting one nonagenarian patient, who was transferred to an inpatient rehabilitation facility, all patients (97.6\%) were discharged from the hospital directly to home or a hotel.

\section{Completeness of Tumor Resection and Need for Additional Treatment}

In 40 of 41 OmniGuide cases, we carried out surgery with the goal of gross-total tumor resection but prioritizing facial nerve function. Based on intraoperative findings, gross-total tumor resection was achieved in 19 cases (47.5\%). Radical subtotal tumor resection, defined as only small scraps of tumor left along the facial nerve, was obtained in 5 cases $(12.5 \%)$. Subtotal or partial resection, defined as anything less, was obtained in 16 cases (40.0\%).

All of these 40 patients underwent one or more postoperative MRI sessions at least 3 months after surgery. On MRI, no nodular enhancement was seen in 24 cases (60.0\%), including 4 of the 5 cases deemed intraoperatively to have been radical subtotal resections and 1 case judged as a subtotal resection. Initial MR images obtained in the other 16 patients revealed residual enhancing masses measuring in the axial plane from $3 \times 2 \mathrm{~mm}$ to $13 \times$ $12 \mathrm{~mm}$.

The median MRI follow-up for all OmniGuide patients was 52.3 months (mean 48.4 months, SD 22.5 months, range 11-81 months). For the 16 patients with residual enhancing masses, the median follow-up was 65 months (range 11-80 months). During follow-up, only 1 patient was found to have a growing residual tumor, which was treated with Gamma Knife radiosurgery (Fig. 3); 12 of the remaining 15 patients have had at least $2 \mathrm{MR}$ images showing stable residual tumors.

One patient, who was 95 years old, underwent planned debulking only. The tumor was a rapidly growing, large mass, and the patient had been rendered wheelchair and nursing-home bound due to symptoms of brainstem compression. Postoperatively, after a brief course of inpatient rehabilitation, the patient returned home and was living independently. Approximately 2 years postoperatively, she again developed symptoms consistent with brainstem compression. She refused further intervention or even imaging and died 30 months after surgery.

\section{Discussion}

Although never having gained widespread acceptance, the use of lasers for intracranial tumor resection has been described numerous times. Various lasers have been used, including $\mathrm{CO}_{2}$, Nd:Yag, Argon, and KTP. . $^{2,14,16,19,24,35}$ More recently, laser energy has been used to thermally ablate intraaxial brain lesions under MRI guidance as a minimally invasive procedure. ${ }^{21,25}$

Of those lasers used for microneurosurgical applications, the $\mathrm{CO}_{2}$ laser has the major advantage of producing a beam within the infrared spectrum $(10.6 \mu \mathrm{m})$. Water is opaque to light at this wavelength, and there is very little penetration of biological tissue, which consists mostly of water. $\mathrm{CO}_{2}$ laser energy can be used very precisely with little thermal spread and little risk to adjacent structures compared with other lasers. ${ }^{29}$ Unfortunately, $\mathrm{CO}_{2}$ lasers cannot be used via fiber-optic transmission, since all possible materials used in the construction of fiber-optic cables are opaque at this wavelength as well.

In 1998, Fink reported efficient propagation of infrared energy through a hollow-core fiber lined with an omnidirectional dielectric mirror. ${ }^{12}$ Among other applications, this led to the development of the OmniGuide $\mathrm{CO}_{2}$ laser system. In this system, the mirror, which consists of alternating layers of high-and low-refractory index materials, is precisely calibrated to guide light generated by the $\mathrm{CO}_{2}$ laser through the flexible fiber in a helium medium. Helium flowing through the fiber also acts as a coolant.

In recent years, the OmniGuide system has become well established for applications in head and neck surgery and gynecological surgery. $1,15,27,33$ Its use has also been reported in the treatment of various types of neurosurgical pathologies, including intracranial tumors, vascular malformations, laminectomy, cord untethering, and corpus callosotomy. ${ }^{4,6-8,18} \mathrm{~A}$ variety of fibers are available for 
different applications, with the Beam Path Neuro-L fiber developed specifically for neurosurgery.

\section{Vestibular Schwannoma Surgery: Safety and Efficacy}

In this series, we have demonstrated that the OmniGuide laser is safe to use in the resection of medium and large vestibular schwannomas via both the TL and RS approaches. There were no complications attributable to the use of the laser. Of course, unlike metal instruments, lasers have the potential to act at a distance. While this allows for no-touch dissection techniques, it also raises the possibility of untoward effects. We took care to always point the laser handpiece toward the tumor's center when working through tissue in the belief that this provided the best assurance of safety. Keeping the depth of the resection cavity filled with saline solution and protecting the facial nerve with cotton patties provided further layers of security. The defocused configuration of the laser exiting the fiber also inherently made injury to distant structures less likely.

Interestingly, we found optimal use of the fiber-based $\mathrm{CO}_{2}$ laser system in microsurgical resection of vestibular schwannomas to be very different from use of the $\mathrm{CO}_{2}$ laser with articulating arms described by Robertson et al. in $1983 .{ }^{26}$ In the earlier study, the laser was used as a focused beam at higher power to cavitate tumors. With the OmniGuide, the laser was used at close quarters, with the dissector nearly touching the tumor, as a cutting tool. At least within the power limitation of $20 \mathrm{~W}$ necessitated by the OmniGuide fiber, we found the laser to be quite limited in vaporization.

While we subjectively found the OmniGuide laser to be highly efficacious in vestibular schwannoma surgery, proving this quantitatively is problematic. Cranial nerve preservation and tumor control compare favorably to those in other reports. ${ }^{3,23,30,31}$ No-touch tumor resection near the porus may help avoid traction on nerves in this particularly delicate location. Use of the $\mathrm{CO}_{2}$ laser may also encourage more complete tumor resection without threat to surrounding structures, leading to smaller residual tumor masses and less chance of regrowth.

We could not statistically demonstrate improvements in ETR or EBL when laser cases were compared directly with controls. The $\mathrm{CO}_{2}$ laser is limited in hemostasis, and it is likely that there was no real benefit in terms of blood loss. On the other hand, it was our strong impression that use of the laser did facilitate more rapid tumor resection. As these were our initial OmniGuide cases, it is possible that there was a learning curve for both the surgeon and operating room staff charged with setting up and running the device. Possibly, at least at first, gains in time of tumor resection were offset by technical time losses. There was also wide variability within both the OmniGuide and control groups.

\section{Utility of the $\mathrm{CO}_{2}$ Laser for Vestibular Schwannoma Surgery}

The utility of the OmniGuide laser must be balanced against its cost. The hospital cost for each fiber is well over $\$ 1000$. We have continued to use the OmniGuide $\mathrm{CO}_{2}$ laser in all cases of very large and giant vestibular schwannomas. In these cases, we believe that there are gains in operative time, and that these gains are probably sufficient to offset the device cost. Certainly, it is our clear perception that the laser reduces the surgeon's fatigue. With very large tumors, the surgeon reaches later stages of the case more energized and ready to face the challenge of facial (and cochlear) nerve dissection.

We also continue to use the laser on an ad hoc basis for tumors that are found intraoperatively to be very firm or otherwise resistant to cutting with scissors. Interestingly, this includes both solid and some cystic tumors. One might conclude that in these cases the usefulness of the $\mathrm{CO}_{2}$ laser is inversely proportional to sharpness of available microscissors. The threat of laser use, and its associated cost, may convince a hospital to maintain sharp scissors in excellent condition on an ongoing basis.

The occurrence of device failures must also be factored into the economic equation of laser use. On several occasions, we experienced burnout of the OmniGuide fiber. In addition to the usual training of operating room personnel in the general use of lasers, staff must also be trained to handle fibers to avoid kinking and to keep dissector tips properly free of debris. Indeed, we have found that more regular use of the laser has reduced the rate of device failure and that failures tend to be more common after longer periods of nonuse or with unfamiliar staff.

\section{Conclusions}

The OmniGuide $\mathrm{CO}_{2}$ laser is a safe and effective tool when used as an adjunct in the resection of medium to large vestibular schwannomas. This device is most useful as a cutting tool and as an aid to piecemeal tumor removal rather than as a tissue vaporizer. The benefit of the OmniGuide laser may be greatest for very large or giant tumors or for very firm tumors resistant to cutting using microscissors.

\section{Acknowledgments}

We thank Karen I. Berliner for statistical analysis and editorial assistance.

\section{References}

1. Barton SE, Gargiulo AR: Robot-assisted laparoscopic myomectomy and adenomyomectomy with a flexible $\mathrm{CO}_{2}$ laser device. J Robot Surg 7:157-162, 2013

2. Beck OJ: Use of the Nd-YAG laser in neurosurgery. Neurosurg Rev 7:151-157, 1984

3. Ben Ammar M, Piccirillo E, Topsakal V, Taibah A, Sanna M: Surgical results and technical refinements in translabyrinthine excision of vestibular schwannomas: the Gruppo Otologico experience. Neurosurgery 70:1481-1491, 2012

4. Browd SR, Zauberman J, Karandikar M, Ojemann JG, Avellino AM, Ellenbogen RG: A new fiber-mediated carbon dioxide laser facilitates pediatric spinal cord detethering. Technical note. J Neurosurg Pediatr 4:280-284, 2009

5. Cerullo LJ, Burke LP: Use of the laser in neurosurgery. Surg Clin North Am 64:995-1000, 1984

6. Choudhri O, Karamchandani J, Gooderham P, Steinberg GK: Flexible omnidirectional carbon dioxide laser as an effective tool for resection of brainstem, supratentorial, and intramedullary cavernous malformations. Neurosurgery 10 (Suppl 1):34-45, 2014

7. Choudhri O, Lober RM, Camara-Quintana J, Yeom KW, 
Guzman R, Edwards MS: Carbon dioxide laser for corpus callosotomy in the pediatric population. J Neurosurg Pediatr 15:321-327, 2015

8. Desai SK, Paulson D, Thompson BJ, Patterson J, Mohanty A: The role of flexible hollow core carbon dioxide lasers in resection of lumbar intraspinal lipomas. Childs Nerv Syst 28:1785-1790, 2012

9. Fasano VA: Observations on the use of three laser sources in sequence $\left(\mathrm{CO}_{2}\right.$-Argon-Nd: YAG) in neurosurgery. Lasers Surg Med 2:199-203, 1983

10. Fayad JN, Friedman RA, Schwartz MD: The retrosigmoid approach, in Friedman RA, Slattery WH, Brackmann JN, Schwartz MS (eds): Lateral Skull Base Surgery: The House Clinic Atlas. New York: Thieme, 2012, pp 38-46

11. Fayad JN, Schwartz MS, Slattery WH, Brackmann DE: Prevention and treatment of cerebrospinal fluid leak after translabyrinthine acoustic tumor removal. Otol Neurotol 28:387-390, 2007

12. Fink Y, Winn JN, Fan S, Chen C, Michel J, Joannopoulos JD, et al: A dielectric omnidirectional reflector. Science 282:1679-1682, 1998

13. Friedman RA, Berliner KI, Bassim M, Ursick J, Slattery WH III, Schwartz MS, et al: A paradigm shift in salvage surgery for radiated vestibular schwannoma. Otol Neurotol 32:13221328,2011

14. Gamache FW Jr, Patterson RH Jr: The use of the potassium titanyl phosphate (KTP) laser in neurosurgery. Neurosurgery 26:1010-1014, 1990

15. Halum SL, Moberly AC: Patient tolerance of the flexible $\mathrm{CO}_{2}$ laser for office-based laryngeal surgery. J Voice 24:750-754, 2010

16. Handa H, Takeuchi J, Yamagami T: Nd-YAG laser as a surgical tool. Neurosurg Rev 7:159-162, 164, 1984

17. House JW, Brackmann DE: Facial nerve grading system. Otolaryngol Head Neck Surg 93:146-147, 1985

18. Hussain NS, Perez-Cruet M: Application of the flexible CO2 laser in minimally invasive laminectomies: technical note. Cureus 8:e628, 2016

19. Jain KK: Lasers in neurosurgery: a review. Lasers Surg Med 2:217-230, 1983

20. Killory BD, Chang SW, Wait SD, Spetzler RF: Use of flexible hollow-core $\mathrm{CO} 2$ laser in microsurgical resection of CNS lesions: early surgical experience. Neurosurgery 66:11871192,2010

21. Lagman C, Chung LK, Pelargos PE, Ung N, Bui TT, Lee SJ, et al: Laser neurosurgery: a systematic analysis of magnetic resonance-guided laser interstitial thermal therapies. J Clin Neurosci 36:20-26, 2017

22. Monsell EM, Balkany T, Gates G, Goldenberg RA, Meyerhoff WL, House JW: Committee on Hearing and Equilibrium guidelines for the evaluation of hearing preservation in acoustic neuroma (vestibular schwannoma). American Academy of Otolaryngology-Head and Neck Surgery Foundation, INC. Otolaryngol Head Neck Surg 113:179-180, 1995

23. Nonaka Y, Fukushima T, Watanabe K, Friedman AH, Sampson JH, Mcelveen JT Jr, et al: Contemporary surgical management of vestibular schwannomas: analysis of complications and lessons learned over the past decade. Neurosurgery 72 (2 Suppl Operative):ons103-ons115, 2013

24. Powers SK, Edwards MS, Boggan JE, Pitts LH, Gutin PH, Hosobuchi Y, et al: Use of the argon surgical laser in neurosurgery. J Neurosurg 60:523-530, 1984

25. Rahmathulla G, Recinos PF, Kamian K, Mohammadi AM, Ahluwalia MS, Barnett GH: MRI-guided laser interstitial thermal therapy in neuro-oncology: a review of its current clinical applications. Oncology 87:67-82, 2014

26. Robertson JH, Clark WC, Robertson JT, Gardner LG, Shea
MC: Use of the carbon dioxide laser for acoustic tumor surgery. Neurosurgery 12:286-290, 1983

27. Ryan M, Kaylie D: CO2 laser myringoplasty: a minimally invasive technique for treating tympanic membrane atelectasis. Otol Neurotol 34:1694-1698, 2013

28. Ryan RW, Spetzler RF, Preul MC: Aura of technology and the cutting edge: a history of lasers in neurosurgery. Neurosurg Focus 27(3):E6, 2009

29. Ryan RW, Wolf T, Spetzler RF, Coons SW, Fink Y, Preul $\mathrm{MC}$ : Application of a flexible $\mathrm{CO}_{2}$ laser fiber for neurosurgery: laser-tissue interactions. J Neurosurg 112:434-443, 2010

30. Samii M, Matthies C: Management of 1000 vestibular schwannomas (acoustic neuromas): the facial nerve-preservation and restitution of function. Neurosurgery 40:684 695, 1997

31. Schwartz MS, Kari E, Strickland BM, Berliner K, Brackmann DE, House JW, et al: Evaluation of the increased use of partial resection of large vestibular schwanommas: facial nerve outcomes and recurrence/regrowth rates. Otol Neurotol 34:1456-1464, 2013

32. Slattery WH: The translabyrinthine approach to the skull base, in Friedman RA, Slattery WH, Brackmann JN, Schwartz MS (eds): Lateral Skull Base Surgery: The House Clinic Atlas. New York: Thieme, 2012, pp 47-66

33. Solares CA, Strome M: Transoral robot-assisted CO2 laser supraglottic laryngectomy: experimental and clinical data. Laryngoscope 117:817-820, 2007

34. Stellar S, Polanyi TG, Bredemeier HC: Experimental studies with the carbon dioxide laser as a neurosurgical instrument. Med Biol Eng 8:549-558, 1970

35. Takizawa T: The carbon dioxide laser surgical unit as an instrument for surgery of brain tumours - its advantages and disadvantages. Neurosurg Rev 7:135-144, 1984

36. Temelkuran B, Hart SD, Benoit G, Joannopoulos JD, Fink Y: Wavelength-scalable hollow optical fibres with large photonic bandgaps for $\mathrm{CO}_{2}$ laser transmission. Nature 420:650-653, 2002

\section{Disclosures}

The authors report no conflict of interest concerning the materials or methods used in this study or the findings specified in this paper.

\section{Author Contributions}

Conception and design: Schwartz. Acquisition of data: Schwartz. Analysis and interpretation of data: Schwartz. Drafting the article: Schwartz. Critically revising the article: both authors. Reviewed submitted version of manuscript: Schwartz. Approved the final version of the manuscript on behalf of both authors: Schwartz.

\section{Supplemental Information \\ Videos}

Video 1. https://vimeo.com/250124133.

\section{Previous Presentations}

Preliminary data were previously presented in abstract form at the AANS Annual Scientific Meeting, Denver, Colorado, April 9-13, 2011.

\section{Correspondence}

Marc S. Schwartz: University of California, San Diego, CA m8schwartz@ucsd.edu. 